\title{
Minimal Surfaces in Nuclear Pasta with the Time-Dependent Hartree-Fock Approach
}

\author{
Bastian Schuetrumpf ${ }^{* \dagger}$ \\ Institut für Theoretische Physik, Universität Frankfurt, D-60438 Frankfurt, Germany \\ E-mail: schuetrumpf@th.physik.uni-frankfurt.de
}

\section{A. Klatt}

Institut für Theoretische Physik, Universität Erlangen-Nürnberg, D-91058 Erlangen, Germany

\section{K. lida}

Department of Natural Science, Kochi University, 2-5-1 Akebono-cho, Kochi 780-8520, Japan

\section{G. E. Schröder-Turk}

Institut für Theoretische Physik, Universität Erlangen-Nürnberg, D-91058 Erlangen, Germany

\section{J. A. Maruhn}

Institut für Theoretische Physik, Universität Frankfurt, D-60438 Frankfurt, Germany

\section{K. Mecke}

Institut für Theoretische Physik, Universität Erlangen-Nürnberg, D-91058 Erlangen, Germany

\section{P.-G. Reinhard}

Institut für Theoretische Physik, Universität Erlangen-Nürnberg, D-91058 Erlangen, Germany

In continuation to the studies of the whole variety of pasta shapes in [1], we present here calculations performed with the Hartree-Fock and time-dependent Hartree- Fock method concerning the mid-density range of pasta shapes: The slab-like, connected rod-like (p-surface) and the gyroidal shapes. On the one hand we present simulations of the dynamic formation of these shapes at finite temperature. On the other hand we calculate the binding energies of these shapes for varying simulation box lengths and mean densities. All of these shapes are found to be at least metastable. The slab shape has a slightly lower energy because of the lack of curvature, but among these three configurations the gyroidal shape is metastable for the widest range in mean density.

52 International Winter Meeting on Nuclear Physics

27 - 31 January 2014

Bormio, Italy

\footnotetext{
*Speaker.

$\dagger$ This work was supported by the BMBF under contract number 05P12RFFTG, DFG for the grant ME1361/11 and by Grants-in-Aid for Scientific Research on Innovative Areas through No. 24105008 provided by MEXT. The calculations have been performed on the cluster of the Center for Scientific Computing of the Goethe-Universität Frankfurt.
} 


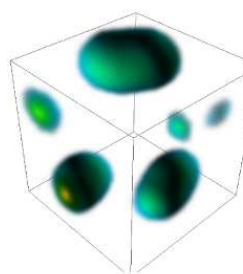

(a) sphere

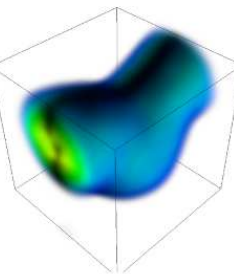

(b) $\operatorname{rod}$

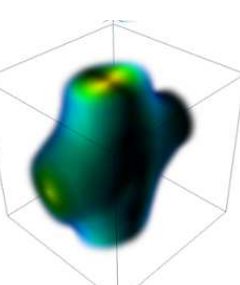

(c) $\operatorname{rod}(2)$

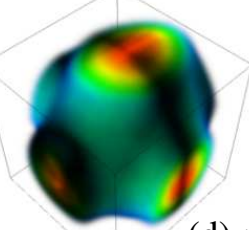

(d) $\operatorname{rod}(3)$
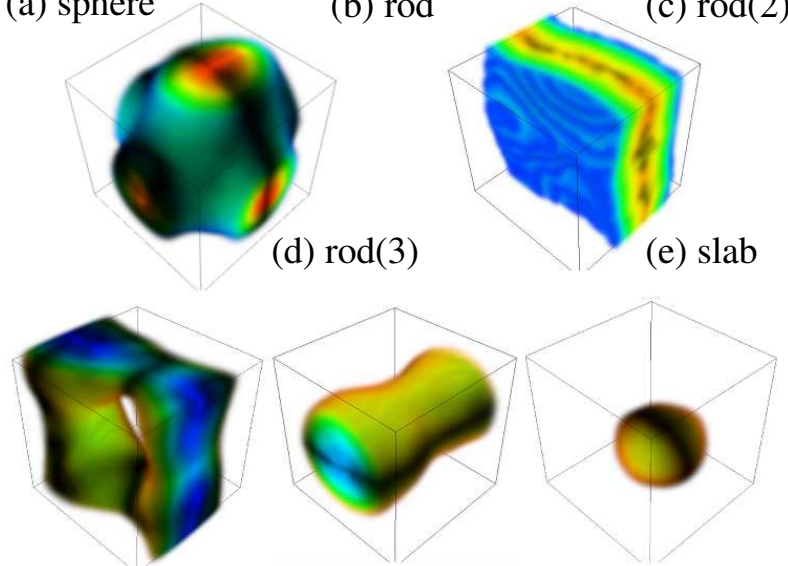

(f) $\operatorname{rod}(2)$ bubble $(\mathrm{g})$ rod bubble $\quad(\mathrm{h})$ sphere bubble

Figure 1: (Color online) Typical shapes of pasta structures. Bubble shape illustrations show gas phase, which is indicated by the color-scale (from $0.03 \mathrm{fm}^{-3}$ (blue/light gray) to $0.12 \mathrm{fm}^{-3}$ (red/dark gray)). This figure is taken from [1].

\section{Introduction}

\subsection{Pasta Matter in Neutron Stars and Supernovae}

Compact stars like neutron stars and supernovae $[2,3]$ contain the very dense nuclear matter. At these densities nuclear matter is not observable in laboratories, but play a crucial role in these scenarios. Normal nuclear matter consists of spherical nuclei which can be slightly deformed because of quantum shell effects to lower their energy. Near the nuclear saturation density $\rho_{0} \approx$ $0.16 \mathrm{fm}^{-3}$ the nuclear matter is a homogeneous quantum Fermi liquid. In between it takes various geometries like rods (spaghetti) and slabs (lasagna) to lower the total surface energy [1] and also more complicated shapes like connected rods (s. Fig. 1). Because of the analogy to Italian pasta, this matter is commonly known as nuclear "pasta". At densities just below the nuclear saturation density the nuclear matter is turned inside out so that the gas phase containing free neutrons take the pasta shapes. These shapes are called pasta bubble shapes.

Most of the pasta phases are liquid crystals [4] and have therefore special features concerning the shear viscosity. As the nuclear pasta layer is located between the inner crust and the outer core in a neutron star, this can affect the rotation between the crust and the core [5]. The special structure of the pasta shapes may also have an influence on the neutrino scattering and therefore affects the thermal properties of a supernova or a neutron star, because the neutrinos can carry an immense part of the energy which is produced during a supernova explosion. 


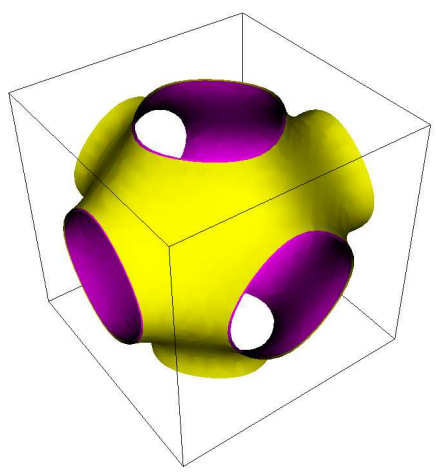

(a) Primitive

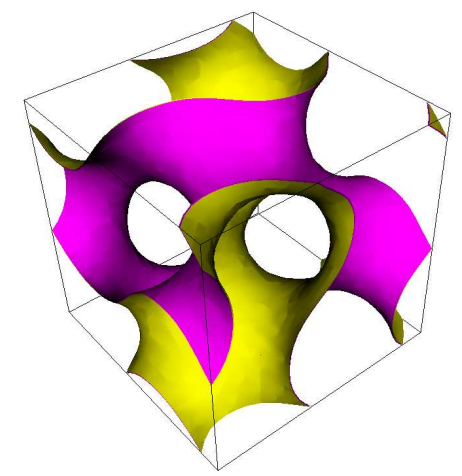

(b) Gyroid

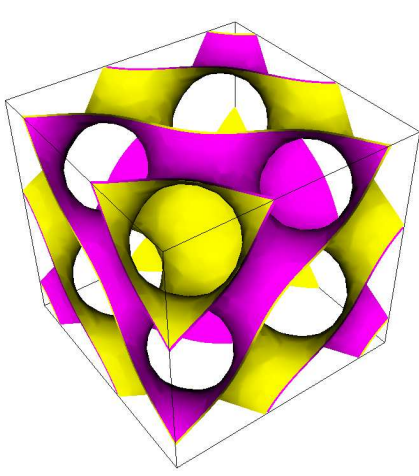

(c) Diamond

Figure 2: (Color online) Nodal approximation of the triply periodic minimal surfaces (see eqs.(1.2), (1.3), and (1.4)). The colors mark the different sides of the surface facing the different domains.

In order to characterize the different pasta shapes with integral measures, the Minkowski functionals are commonly used. The scalar Minkowski functionals are proportional to the volume $V$, the surface area $A$, the integral mean curvature $\int H \mathrm{~d} A$ and the Euler characteristic $\chi=\int K \mathrm{~d} A$ with the mean curvature $H=\left(\kappa_{1}+\kappa_{2}\right) / 2$ and the Gaussian curvature $K=\kappa_{1} \kappa_{2}$ with the two principal curvatures $\kappa_{1}$ and $\kappa_{2}$. Additionally we introduce a tensorial Minkowski functional $[6,7]$ to describe the pasta shapes, namely the distribution of the normal vectors on the surface. The eigenvalue ratio $\beta_{1}^{0,2}$ of this interface tensor $W_{1}^{0,2}$ (see [7] for the definition) describes the anisotropy of the structures. If $\beta_{1}^{0,2}=1$, the structure is perfectly isotropic, if the value is below one, the structure is anisotropic.

\subsection{Minimal Surface Shapes}

Recently triply periodic minimal surface (TPMS) shapes have come into focus, especially the Gyroid $(G)$ was investigated $[8,9]$ in the connection with pasta matter. Gyroids were already found in solid biological systems, in di-block polymers, and in inverse bicontinous phases in lipid water systems (s. e.g. Refs. $[10,11,12])$. An interesting feature is that the Gyroid network contains screw axes. Also the rod(3) structure shown in Fig. 1(d) is topologically equivalent to the Primitive (P) TPMS. Together with the Diamond (D) these three surfaces (s. Fig. 2) form a group of TPMS which are related by the Bonnet transformation [13]. Note that the Bonnet transformation is a transformation between these shapes bending the surfaces isomorphically, but has no physical relevance, because all intermediate surfaces have self-intersections. All these surfaces have minimal surface area under different boundary conditions. This is equivalent to $H=0$ at each point of the surface. The Bonnet transformation relates the cubic periodic lengths of the TPMS such that all metric and curvature properties are unchanged via $a_{P} / a_{G}=0.81$ and $a_{D} / a_{G}=1.27$.

The introduced TPMS divide the space into two half-spaces (domains) with equal volume. Since the pasta shapes can cover an arbitrary volume fraction $u$ the TPMS idea has to be expanded to the constant mean curvature (CMC) surfaces. With this definition the volume fractions can be varied keeping the topology of the surface. The surfaces can be approximated with the nodal 
approximation. Together with the slab it yields

$$
\begin{aligned}
\phi_{S} & =\phi_{0} \cdot(\cos x) \\
\phi_{P} & =\phi_{0} \cdot(\cos x+\cos y+\cos z) \\
\phi_{G} & =\phi_{0} \cdot(\cos x \sin y+\cos y \sin z+\cos z \sin x) \\
\phi_{D} & =\phi_{0} \cdot(\cos x \cos y \cos z+\cos x \sin y \sin z+\sin x \cos y \sin z+\sin x \sin y \cos z)
\end{aligned}
$$

with a parameter $\phi_{0}$. The TPMS are approximated by $\phi_{i}=0$ with $i \in\{S, P, G, D\}$. By tuning the parameter $\phi_{i}$ the volume fraction $u$ can be varied. Regarding the rough voxelization of the grid the calculations are performed on, the nodal approximation is sufficient.

There can be different kinds of TPMS structures. The single structures are characterized by the matter covering one domain. The topology of the two domains is equal, the domains only can have different volume fractions. The single $\mathrm{P}$ structure has an Euler characteristic of $\chi_{P}=-2$, for $\mathrm{G} \chi_{G}=-4$, and for $\mathrm{D} \chi_{D}=-8$. These conditions are not sufficient to identify these structures. There can be topologically different structures with the same Euler characteristic.

There are two double structures. One is composed of two TPMS structures which are bounded by two CMC surfaces with mean curvature $\pm H$. They are separated by the so-called matrix phase. The other double structure is characterized by the matter filling out the matrix phase. In the following we concentrate on the single structures, because the double structures were not found to be metastable under the assumed conditions.

\subsection{The Hartree-Fock and Time-Dependent Hartree-Fock Method}

The TPMS structures in pasta matter are investigated with the Hartree-Fock and timedependent Hartree-Fock method which are both well established methods for nuclear ground state calculations or reaction dynamics simulations, respectively. For both methods the wave functions are restricted to a single slater determinant. For the Hartree-Fock method the variational principle

$$
\delta\langle\Psi|\hat{H}-E| \Psi\rangle=0
$$

is solved. This is done iteratively until convergence and with it the ground state is reached. The damped gradient step with kinetic energy inversion is utilized here.

For the time-dependent Hartree-Fock method the time-dependent variational principle

$$
\delta\left\langle\Psi\left|\hat{H}-i \hbar \frac{\partial}{\partial t}\right| \Psi\right\rangle=0
$$

is solved. This can be done by applying the time evolution operator to the single particle wave functions for finite time steps. In this case we use time steps of $\Delta t=0.1 \frac{\mathrm{fm}}{\mathrm{c}}$.

All calculations are performed on an equidistant 3D grid with a grid spacing of $\Delta r \in$ $[0.875 \mathrm{fm}, 1.125 \mathrm{fm}]$ and an even number $N_{X}=N_{Y}=N_{Z}=16-24$ of grid points. Thus boxes result which have a box length of $15-26 \mathrm{fm}$. We take periodic boundary conditions. For a detailed description of the methods and the implementation see [14]. 


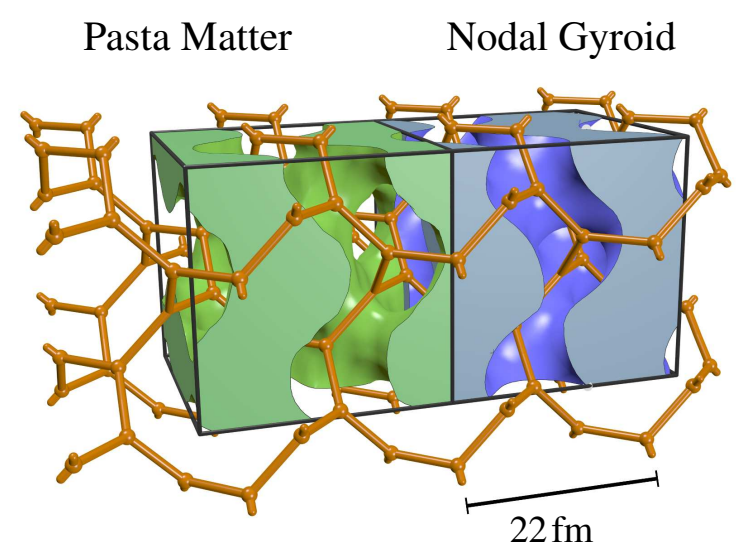

Figure 3: (Color online) Gyroidal pasta shape: the green structure on the left shows the density distribution of of nuclear pasta matter computed with TDHF for an average density of $0.06 \mathrm{fm}^{-3}$ and box length $a=22 \mathrm{fm}$. Shown is the surface of half maximum density with the solid volume representing densities above this value. The blue structure on the right shows the nodal approximation of a gyroid CMC surface at the same volume fraction. Orange bars illustrates the gyroid network in the void phase of both the pasta shape and the nodal approximation, showing that they are indeed homotopic. Black frames are guides to the eye, of size $1.25 a$ the cubic lattice parameter. This figure is taken from [15].

\section{Results}

\subsection{Time-Dependent Calculations}

In earlier calculations pasta shapes were found which are topologically equal to $\mathrm{P}$ structures (s. Fig. 1). These calculations were performed with a box length of $16 \mathrm{fm}$. Assuming that the nuclear pasta Hamiltonian can be described in terms of the curvature, the Bonnet transformation predicts, that $\mathrm{G}$ structures may appear at larger box lengths. Therefore we performed calculations with box lengths of $20-24 \mathrm{fm}$ with random initial conditions. To that end $\alpha$-particles are distributed on the grid randomly and additional background neutrons are distributed as the lowest plane wave states. The proton fraction for all calculations is set to $X_{P}=1 / 3$. For each box length ten calculations were performed with a mean density of $0.06 \mathrm{fm}^{-3}$.

In order to identify the $G$ shapes, a preselection with the Euler characteristic is done. To identify G clearly, a gyroid network is used (s. Fig 3). If this network fits perfectly into the voids, the structure is gyroidal.

The Euler characteristics for the resulting shapes are shown in Fig. 4. We marked the G structures explicitly by shaded areas. Not all of the shapes which have an Euler characteristic of -4 are $\mathrm{G}$ shapes. Furthermore the Euler characteristics decrease for increasing box lengths which indicates more labyrinthlike structures. As nuclear matter has an intrinsic length scale and thus tends to build up connections of a certain size, more rodlike connections can be built in bigger boxes.

In sum we find seven $G$ shapes. These shapes have the topology of $G$ but all of them are strongly anisotropic. $\beta_{1}^{0,2}$ takes values down to 0.5 , but their topology remains stable in the the time-evolution. 


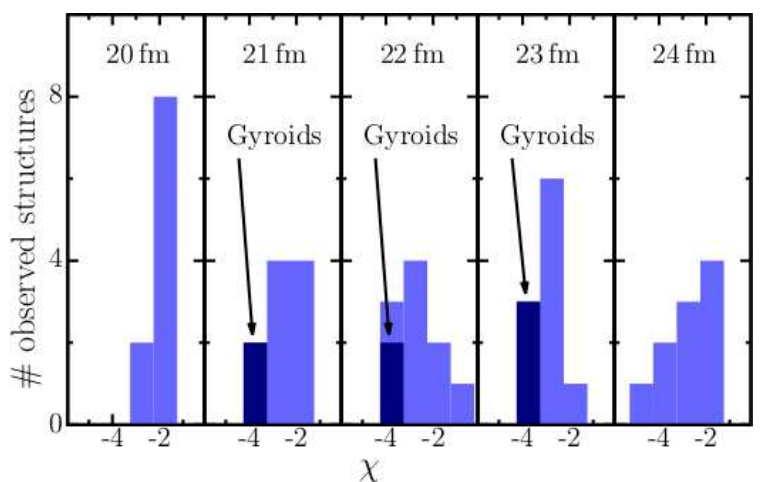

Figure 4: (Color online) Histograms of the resulting Euler characteristic $\chi$ for different box lengths $20-24 \mathrm{fm}$ of the dynamic, randomly initialized calculations. The shaded area marks the identified gyroidal structures. This figure is taken from [15].

\subsection{Ground State Calculations}

Additionally we perform ground state calculation in order to test the (meta-)stability of the TPMS. To that end we initialize the calculations only with plane wave wave-functions again with a proton fraction of $X_{P}=1 / 3$. For the first 1000 iterations we add an additional potential. We perform for the different values of mean density and the corresponding volume fractions $u$ calculations with the potentials $\phi_{i}$ from the equations $1.1,1.2,1.3$, and 1.4 with $\phi_{0}=10 \mathrm{MeV}$. These potentials force the nuclear matter to take the shapes of the TPMS or the slab, which is expected to appear at the same densities and thus is competitive to the TPMS, respectively. The calculations are performed for further 9000 iteration without the potential. If the pasta structures stays topologically equal to the structure the matter was forced to in the first 1000 iterations, we regard this state as a metastable state.

As the nuclear pasta has a smooth transition between the void and the nuclear matter the value for $u$ is calculated here with the Gibbs dividing surface method. All of the nuclear matter is expected to be in the liquid phase with a constant density. Thus the volume fraction is

$$
u=\frac{\rho}{\rho_{l}}
$$

where $\rho_{l}$ is the maximum density on the grid and $\rho$ is the mean density.

In Fig. 5 the resulting binding energies per nucleon are plotted. Note that a larger binding energy refers to a stronger binding. Only very few D structures were stable at the chosen parameters. These were left out for simplicity. We expect $\mathrm{D}$ structures to be meta-stable at larger boxes with a box length larger than $26 \mathrm{fm}$ which was computationally not feasible. All resulting G shapes are only slightly anisotropic with $\beta_{1}^{0,2}>0.75$.

$\mathrm{G}$ and $\mathrm{P}$ structures show a strong dependence on the box length. $\mathrm{P}$ has a maximum binding energy for $a=22 \mathrm{fm}$. Taking the Bonnet transformation the maximum binding energy for G should be reached at a box length of $27 \mathrm{fm}$. Thus the calculated values for $26 \mathrm{fm}$ can serve as a good approximation for the maximum binding energies. The slab does not show a strong scaling with the box length. The maximum binding energies for the slab is slightly larger than the maximum binding energies for the TPMS, except for the highest volume fraction, but at these high densities 


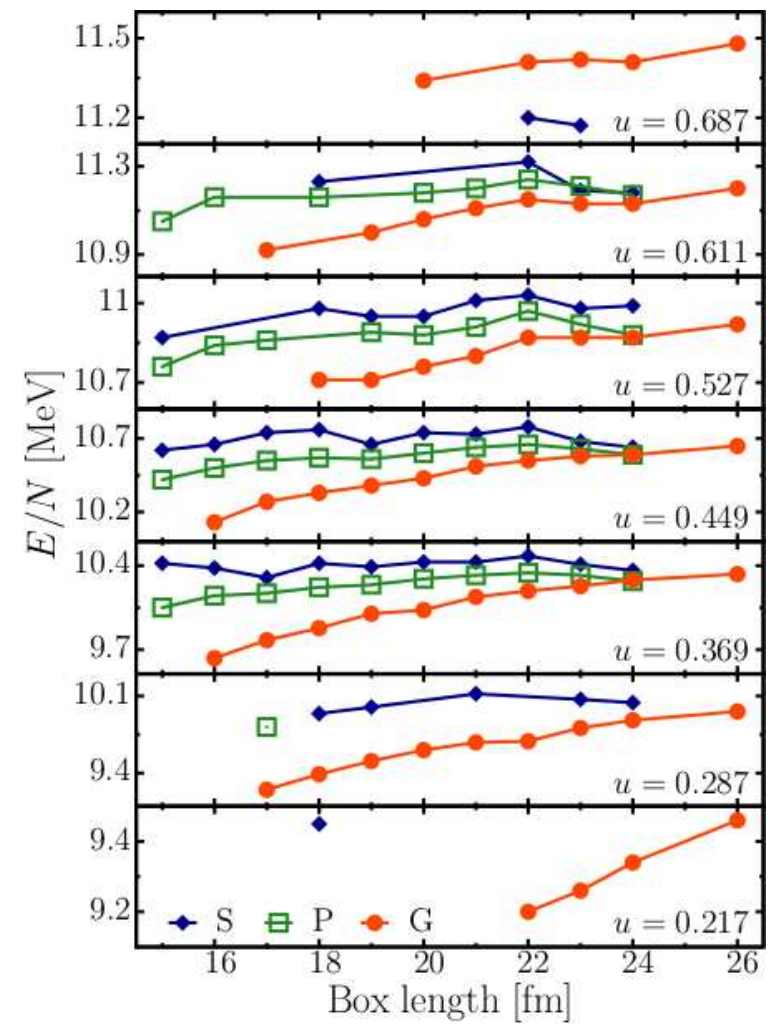

Figure 5: (Color online) Binding energies per nucleon $E / N$ for the metastable ground states for different volume fractions. G (gyroid, dots), $\mathrm{P}$ (primitive, squares) and $\mathrm{S}$ (slab, diamonds) denotes the potential which was added to the mean field. The states shown in this plot remained topologically stable for the 9000 iterations. Note that higher values of the energy correspond to more tightly bound and hence more favorable solutions. This figure is taken from [15].

the rod(2) bubble shape has larger binding energies anyway. This difference is very small. For that reason the pasta matter in cold neutron stars or hot supernovae at these densities might consist of mixed pasta phases.

Furthermore the dynamic calculations described above are cooled with static Hartree-Fock to investigate the anisotropy of the shapes and the influence on the binding energies. Only three of the seven dynamic shapes remain gyroidal. $\beta_{1}^{0,2}$ increases slightly but taking a shape with $a=22 \mathrm{fm}$ as an example does not exceed 0.65. Comparing this shape to the ground state from the plane wave initialized calculations shows, that the binding energies of the anisotropic $\mathrm{G}$ is not so much different from the isotropic. The difference is $\Delta E / A=0.02 \mathrm{MeV}$. As the scalar Minkowski measures are equal it seems, that the binding energy depends on the scalar values like volume, surface area and mean curvature.

\section{Conclusion}

We have shown with the Hartree-Fock and time-dependent Hartree-Fock method, that gyroids are competitive to slabs in nuclear pasta. G, although very involved structures with a certain hand- 
edness are built from random initial conditions of $\alpha$-particles and free neutrons. These structures are anisotropic but still gyroidal and can be identified by graphical analysis with the G network.

Furthermore we showed that the TPMS structures initialized with plane waves and a guiding potential are metastable. The $\mathrm{P}$ structures have a maximum binding energy for $A=22 \mathrm{fm}$. The $\mathrm{G}$ structures should have a maximum binding energy at $a=27 \mathrm{fm}$. Both have slightly lower binding energies and therefore may be slightly less favored in neutron stars and supernovae.

Anisotropic dynamic structures stay anisotropic under cooling. This is not crucial for the binding energy.

\section{References}

[1] B. Schuetrumpf, M. A. Klatt, K. Iida, J. A. Maruhn, K. Mecke, and P.-G. Reinhard, Phys. Rev. C 87, 055805 (2013)

[2] H. A. Bethe, Rev. Mod Phys. 62, 801 (1990)

[3] H. Suzuki, Physics and Astrophysics of Neutrinos, Springer, Tokyo 1994.

[4] C. Pethick and A. Potekhin, Phys. Lett. B 427, 7 (1998)

[5] J. A. Pons, D. Viganò N. Rea, Nat. Phys. 9, 431-434 (2013)

[6] G. E. Schröder-Turk et al., Adv. Matter. 23, 2535 (2011)

[7] G. E. Schröder-Turk, W. Mickel, S. C. Kapfer, F. M. Schaller, B. Breidenbach, D. Hug and K. Mecke, New Journal of Physics 15, 083028 (2013)

[8] K. Nakazato, K. Oyamatsu, and S. Yamada, Phys. Rev. Lett. 103, 132501 (2009)

[9] K. Nakazato, K. Iida, and K. Oyamatsu, Phys. Rev. C 83065811 (2011)

[10] K. Michielsen and D. Stavenga, J. R. Soc. Interface 5, 85 (2008)

[11] D. Hajduk, P. Harper, S. Gruner, C. Honeker, G.Kim, E. Thomas, and L. Fetters, Macromolecules 27, 4063 (1994)

[12] K. Larsson J. Phys. Chem. 93, 7304 (1989)

[13] S. T. Hyde, S. Andersson, K. Larsson, Z. Blum, T. Landh, S. Lidin, and B. Ninham, The Language of Shape, 1st ed., Elsevier Science, Amsterdam, (1997)

[14] J. A. Maruhn, P.-G. Reinhard, P. D. Stevenson, and A. S. Umar, Comp. Phys. Comm. to appear (2014) arXiv:1310.5946

[15] B. Schuetrumpf, M. A. Klatt, K. Iida, G. E. Schröder-Turk, J. A. Maruhn, K. Mecke, and P.-G. Reinhard, Appearance of the Gyroid Network Phase in Nuclear Pasta Matter submitted to PRL (2014) arXiv:1404.4760 